\title{
Antimicrobial adjuvants- an innovative strategy for handling antimicrobial resistance displayed by microbes
}

\begin{abstract}
Human and animal health care workers around the world are sounding the alarm for the development of new antibiotics to counter the ever-growing menace of multidrug-resistant pathogenic bacteria. However, there are increasing numbers of reports regarding extremely drug-resistant bacteria for which there are limited or no therapeutic options. So far the drug discovery has failed to keep pace with the rapidly evolving microbes which has resultantly lead to innovation gap related to development and marketing of new antibiotics. Moreover, microbial resistance towards antimicrobials is a way complex and multifaceted mechanism. Nevertheless, many strategies may be employed to minimize the impact and emergence of resistance. Use of antimicrobial adjuvants are one of such effective strategies. These adjuvants are chemically active moieties that have slight to no antibiotic action but may block antimicrobial resistance or boost action of antimicrobial. They are therefore administered along with antimicrobials and may be divided broadly into two important groups: The Class I agents having direct action on microbe, whereas Class II agents having direct action on host defense mechanisms to potentiate antimicrobial action. Antimicrobial adjuvants are a mean to suppress the emergence of bacterial resistance as well as rescue the antimicrobial activity of present drugs in a cost-effective manner, presenting an orthogonal approach to new antibiotic discovery.
\end{abstract}

Keywords: methicillin, gentamicin, vancomycin, imipenem, Levofloxacin, linezolid, HGT, AST
Volume 5 Issue 4 - 2017

\author{
Zaytoon Zaheer, Sajjad Ur Rahman, lqra \\ Zaheer, Tayyaba Younas, Ghazanfar Abbas, \\ Tean Zaheer \\ Faculty of Veterinary Science, University of Agriculture \\ Faisalabad, Pakistan
}

Correspondence: Zaytoon Zaheer, Lecturer, Institute of Microbiology, University of Agriculture Faisalabad, Pakistan, Email zaytoonzaheer@gmail.com

Received: August 10,2017 | Published: October 30, 2017

\section{Introduction}

Revolutionization in the world of medicine can be hailed to ground breaking discovery of Penicillin by Alexander Flaming and was successfully used to treat infections of world war II soldiers until its resistance emerged. Later, scientists developed a big group of antimicrobials including tetracycline, erythromycin, methicillin, gentamicin, vancomycin, imipenem, levofloxacin, linezolid and were potentially effective enough to deal with microbes and their associated infections. However, irrational usage of these antimicrobials has led to exhibition of resistance by microbes particularly to the routinely used antimicrobials. It is noteworthy that during the end of 1960s up to the early $1980 \mathrm{~s}$, the medicine industry introduced a pool of new antibiotics to deal with the resistance, but soon after that antibiotic pool was observed to dry up and only fewer new molecules were introduced. This is the reason that the era between 1960 till 2000 is regarded as innovation gap for antimicrobial discovery. ${ }^{1}$

\section{Mechanism of bacterial resistance}

Bacterial resistance towards antimicrobials is generally classified into three main categories: intrinsic, acquired and adaptive mode of resistance. ${ }^{2}$ Intrinsic resistance manifested by bacteria to antibiotics is not associated to antibiotic induced selection pressure but it is mainly related to specific properties of bacteria itself. The presence of lipopolysaccharides in Gram-negative bacteria for example act as a barrier for antimicrobials attempting to enter the bacterial cell. Furthermore, bacteria possessing drug efflux pumps can perform pumping of antibiotics outside the cell. Acquired mode of resistance happens upon mutational activities in chromosome orupon insertion of new genetic material (plasmid DNA, transposons, episomes and integrons) in the existing genome through horizontal gene transfer (HGT).This type of resistance is passed on to the daughter bacterial cells producing resistant cells. Along with discovery of new antimicrobials, methods of bacterial resistance have also co-evolved in a more rapid manner. ${ }^{3}$ Adaptive mode of resistance involves a brief rise in capability of bacteria to endure the presence of an antibiotic in an effective way, mostly due to alteration of genes and/or variation in expression of proteins prompted in response to ecological conditions (i.e. nutrient concentration, stress, state of growth, sub inhibitory antibiotic levels). This type of resistance is not stable and hence not transferred to progeny because it can be reversed upon removal of resistance prompting factor. The combination of these resistance mechanisms displayed by microbes has provoked the emergence of pathogens which are multidrug-resistant and consequently are a significant concern for human and animal health workers. ${ }^{4}$

\section{Antibiotic resistance associated losses}

Antibiotic resistance has not only made the treatment of infections difficult but has also resulted in a lot of associated losses including increase in individual's treatment cost, disturbance in natural eco-system by introducing of resistant microbes, burden on national economy, prolonged hospital stays, frequent doctor visits, psychological upsets, increase in morbidity, mortality and case fatality and may also compromise the success of major surgeries and cancer chemotherapy. ${ }^{5}$ 


\section{Methods to deal with antibiotic resistance}

There are many strategies to handle resistance displayed by various microbes towards a large repertoire of antimicrobials including routine diagnostic testing to precisely identify the microbe, antimicrobial susceptibility testing (AST) of clinical samples, monitoring of clinical responses to treatment, newer antibiotic development, controlled usage of antimicrobials in food animals, antibiotic resistance awareness education for public, discriminate use of antibiotic in prescription, vaccine development for bacterial infections, use of antimicrobial peptides or bacteriophages for treating resistant bacterial infections. ${ }^{6}$ Nevertheless, the process of discovering newer antimicrobial molecules was considered as an effective way to deal with the deficiencies faced by the existing compounds. However, the efforts for development as well as marketing the approval of newer antibiotic molecules have failed to keep pace with the ever increasing and evolving threat to public health in terms of bacterial resistance. Hence, scientists have always focused to deal the resistance of microbes through different strategies. ${ }^{7,8}$

\section{Antimicrobial adjuvants and efforts for their discovery}

An amazing and promising alternative to development of newer antimicrobials is exploring the enhancers of existing drug arsenal, a relatively cost effective solution. The enhancers of antimicrobial action are regarded as antibiotic adjuvants. They are chemically active moieties, predominantly possessing little or no-antibiotic action, so upon combination with antimicrobials they may boost the antibiotic action. The combination of synergistic antimicrobials is also classified under antibiotic adjuvants. The action of antibiotic adjuvants may occur by causing reversion of the bacterial resistance strategies in obviously sensitive microbe or may cause sensitization of intrinsically resistant microbial strains. The identification of newer molecules which may act as antimicrobial adjuvants is considered the need of the hour. ${ }^{9}$ A research was conducted by Kalan and Wright, where they used Escherichia coli as model organism and studied different molecules as adjuvants for novobiocin. During their project four molecules that enhance the antimicrobial action of novobiocin as well as action of other antimicrobials acting on 'Gram-positive' microbes were also tested for their efficacy to treat $E$. coli induced infections. Almost all identified chemically active moieties were observed to alter the morphology of bacterial cell through inhibiting the proteins of cytoskeleton and/or biosynthesis of peptidoglycan, acting synergistically with antibiotics used in research. It was concluded that the bacterial cell shape alteration is likely to disturb the drug influx/ drug efflux machinery in Gram-negatives enabling the accumulation of routine/ resistance excluded antimicrobials. This study provided an insight of an amazing strategy for combating intrinsic antimicrobial resistance displayed by Gram negative microbes and may also assist in the discovery of newer therapies which may boost the action of already discovered antimicrobials. Four antimicrobial adjuvants identified during this work were found to potentiate the antimicrobial spectrum of action by altering an important physiological activity exhibited by bacteria, however boosting activity of such compounds may also be induced via: (i) enhancement of drug uptake through bacterial plasma membrane; (ii) direct blockade in drug efflux mechanism; (iii) change in physiological pathways of resistance exhibiting strains; and (iv) inhibition of antimicrobial resistance elements (such as scattering of biofilms into freely living planktons, in turn being more susceptible to antibiotics). ${ }^{10}$ Taylor along with his colleagues did a research study to identify and highlight moieties that have ability to potentiate action of existing antibiotics which are in common practice for Gram-positive bacterial infectionshaving however, little or negligible effects on infections caused by Gram negatives. ${ }^{11}$

\section{Types of antimicrobial adjuvants and their mode of action}

Antibiotic adjuvants are broadly classified in two main classes based on their targets: Class I antibiotic adjuvants work along with the antibiotics upon bacterial cell targets, whereas Class II antibiotic adjuvants may enhance the antimicrobial activity in the host. Class I antibiotic adjuvants are further differentiated because of their mechanism of action and include Class I.A molecules that directly inhibit the antibiotic resistance (by inactivation of resistance conferring enzymes, altering the drug efflux pump mechanism, or by altering their targets) and are currently the only antibiotic adjuvants in clinical usage. Class I.B molecules enhance the antibiotic action by evading intrinsic antimicrobial resistance mechanisms including bacterial metabolic pathway evasion or physiological mechanisms other than inducing direct inhibition of resistance elements. Moreover, Class II antibiotic adjuvants do not impact bacterial cell directly but operate on host defense mechanisms to potentiate antibiotic activity. Class I. B as well as Class II antibiotic adjuvants are not yet approved by regulatory authorities, but are used in preclinical trials. ${ }^{12}$

\section{Examples of class I.A antibiotic adjuvant}

Zerbaxa (ceftolozane/tazobactam), is a combination that is particularly effective against Pseudomonas, and was approved by food and drug administration authority (FDA) during 2014. Carbavance, is a combination of vaborbactam- an antibiotic adjuvant for meropenem, and has received recently a fast-track status from FDA. ${ }^{13}$ Examples of class I.B antibiotic adjuvant

Two important examples of this include loperamide for Gram negative bacteria, combination of PA $\beta \mathrm{N}$ (phenylalaninearginine $\beta$-naphthylamide) with fluoroquinolone antibiotic such as levofloxacin. However, challenges in terms of PA $\beta N$ toxicity other has prevented their clinical usage. ${ }^{9}$

\section{Examples of class II antibiotic adjuvant}

Immunomodulatory antimicrobial peptides such as LL-37 have been known for long to enhance the antimicrobial action of innate immunity, and are under, clinical trials. ${ }^{14,15}$ Moreover, screening of microbial natural product extracts to enhance the lytic activity of macrophage against Streptococcus mutans has identified a neutral compound called streptazolin, which may be used as antibiotic adjuvant. However, there is a dire need to create balance regarding immune modulation to prevent over activation of host immune system.

\section{Conclusion}

Antibiotic resistance is a menace for medicine today. Newer drug candidates are difficult to be discovered and brought to the market within a short period hence resulting in a never-increasing gap between the evolving clinical needs and drug discovery. However, antimicrobial adjuvants offer relatively an orthogonal approach to newer antibiotic discovery strategy. These chemically active compounds may enhance and ensure the preservation of action by our existing drug repertoire. Consequently, the impact of antibiotic resistance may be minimized and slowed down (though can be never eliminated). 


\section{Acknowledgements}

None.

\section{Conflict of interest}

The author declares no conflict of interest.

\section{References}

1. Spellberg B, Gilbert DN. The future of antibiotics and resistance: a tribute to a career of leadership by John Bartlett. Clin Infect Dis. 2014;59(Suppl 2):71-75.

2. Alekshun MN, Levy SB. Molecular mechanisms of antibacterial multidrug resistance. Cell. 2007;128(6):1037-1050.

3. D'Costa VM, King CE, Kalan L, et al. Antibiotic resistance is ancient. Nature. 2011;477(7365):457-461.

4. Poole K. Stress responses as determinants of antimicrobial resistance in Gram-negative bacteria. Trends Microbiol. 2012;20(5):227-234.

5. Sengupta S, Chattopadhyay MK, Grossart HP. The multifaceted roles of antibiotics and antibiotic resistance in nature. Front Microbiol. $2013 ; 4: 47$.

6. Livermore DM. Minimising antibiotic resistance. Lancet Infect Dis. 2005;5(7) 450-459.
7. Dye C. Doomsday postponed? Preventing and reversing epidemics of drug-resistant tuberculosis. Nat Rev Microbiol. 2009;7(1):81-87.

8. Hayashi Y, Paterson DL. Strategies for reduction in duration of antibiotic use in hospitalized patients. Clin Infect Dis. 2011;52(10):1232-1240.

9. Ejim L, Farha MA, Falconer SB, et al. Combinations of antibiotics and non antibiotic drugs enhance antimicrobial efficacy. Nat Chem Biol. 2011;7(6):348-350.

10. Kalan L, Wright GD. Antibiotic adjuvants: multicomponent antiinfective strategies. Expert Rev Mol Med. 2011;13:511-517.

11. Taylor PL, Rossi L, De Pascale G, et al. A forward chemical screen identifies antibiotic adjuvants in Escherichia coli. ACS Chem Biol. 2012;7(9):1547-1555.

12. Worthington RJ, Melander C. Combination approaches to combat multidrug-resistant bacteria. Trends Bio-technol. 2013;31(3):177-184.

13. Gill EE, Franco OL, Hancock RE. Antibiotic adjuvants: diverse strategies for controlling drug-resistant pathogens. Chem Biol Drug Des. 2015;85(1):56-78.

14. Hancock RE, Nijnik A, Philpott DJ. Modulating immunity as a therapy for bacterial infections. Nat Rev Microbiol. 2012;10(4):243-254.

15. Perry JA, Koteva K, Verschoor CP, et al. A macrophage-stimulating compound from a screen of microbial natural products. $J$ Antibiot (Tokyo). 2015;68(1):40-46. 